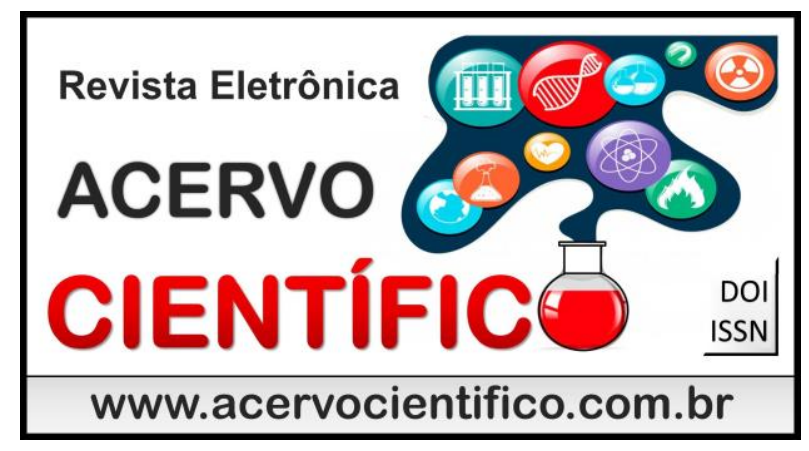

\section{REVISÃO DE LITERATURA}

Recebido em: 4/2019

Aceito em: 5/2019

Publicado em: 6/2019

\title{
Pancreatite crônica - fisiopatologia e tratamento: uma revisão de literatura
}

Chronic pancreatitis - pathophysiology and treatment: a review of literature

Pancreatite Crónica - fisiopatología y tratamiento: una revisión de literatura

Adriane Ribeiro Costa ${ }^{1}$, Bianca Barros Branco ${ }^{1 *}$, Ryan Jorge Amorim², Ana Paula Santos Oliveira Brito ${ }^{3}$.

Resumo: O objetivo deste estudo foi realizar uma revisão de literatura, entre o ano de 2014 e 2018, no que tange à fisiopatologia e ao tratamento da pancreatite crônica. Diante da análise dos estudos dessa revisão, pôde-se observar que a fisiopatologia da pancreatite crônica ainda permanece mal compreendida. A pancreatite crônica alcoólica -que é a mais comum - revelou-se como principal alvo de interesse da comunidade científica atualmente. Quanto ao tratamento da pancreatite crônica notou-se que o mesmo é essencialmente baseado no controle da dor e, quando necessária, na abordagem endoscópica e na intervenção cirúrgica. Percebeu-se, por fim, a relevante necessidade de novas linhas de pesquisa sobre 0 assunto a fim de se esclarecer, de forma consistente, a fisiopatologia da pancreatite crônica e de que maneira cada parte da terapêutica influencia na melhora clínica do paciente portador dessa afecção.

Palavras-chave: Pancreatite crônica, Fisiopatologia, Terapia.

\begin{abstract}
The objective of this study was to perform a review of the literature, between the year 2014 and 2018, regarding the pathophysiology and treatment of chronic pancreatitis. Considering the analysis of the studies of this review, it could be observed that the pathophysiology of chronic pancreatitis still remains poorly understood. Chronic alcoholic pancreatitis - which is the most common - has proved to be the main target of interest for the scientific community today. Regarding the treatment of chronic pancreatitis, it was observed that it is essentially based on pain control and, when necessary, on endoscopic approach and surgical intervention. Finally, the relevant need for new lines of research on the subject was finally understood in order to consistently clarify the pathophysiology of chronic pancreatitis and how each part of the therapy influences the clinical improvement of the patient with this condition.
\end{abstract}

Keywords: Pancreatitischronic, Physiopathology, Therapy.

\footnotetext{
${ }^{1}$ Discentes do Centro Universitário Metropolitano da Amazônia (UNIFAMAZ), Belém-PA.

*E-mail: biancabarrosb@hotmail.com

${ }^{2}$ Graduado em Medicina pela Universidade do Estado do Pará (UEPA), Belém-PA.

${ }^{3}$ Graduada em Medicina pela Universidade Federal do Pará (UFPA), Belém-PA.
} 
Resumen: El objetivo de este estudio fue realizar una revisión de literatura, entre el año 2014 y 2018, en lo que se refiere a la fisiopatología y al tratamiento de la pancreatitis crónica. Ante el análisis de los estudios de esta revisión, se pudo observar que la fisiopatología de la pancreatitis crónica aún permanece mal comprendida. La pancreatitis crónica alcohólica -que es la más común - se ha revelado como principal objetivo de interés de la comunidad científica actualmente. En cuanto al tratamiento de la pancreatitis crónica se notó que el mismo es esencialmente basado en el control del dolor y, cuando es necesario, en el abordaje endoscópico y en la intervención quirúrgica. Se percibió por fin la relevante necesidad de nuevas líneas de investigación sobre el tema a fin de esclarecer de forma consistente la fisiopatología de la pancreatitis crónica y de qué manera cada parte de la terapéutica influye en la mejora clínica del paciente portador de esa afección.

Palabras clave: Pancreatitis Crónica, Fisiopatología, Terapia.

\section{INTRODUÇÃO}

A pancreatite crônica é responsável por morbidade e custos médicos substanciais (FELDMAN M et al, 2014). Essa patologia se caracteriza pela substituição progressiva e irreversível do parênquima pancreático normal por tecido fibroso. Os avanços no conhecimento da etiologia da PC permitiram desenvolver o sistema de classificação TIGAR-O. Os fatores de risco e etiológicos definidos por este sistema de classificação são: tóxicos-metabólicos (álcool, tabaco, hiperlipidemia, hipercalcemia, insuficiência renal crónica, toxinas e medicação); idiopático; genéticos (mutações nos genes PRSSI, CFTR ou SPINKI); autoimunidade; pancreatite aguda severa e recorrente; obstrutivos (pâncreas divisum, quistos, obstrução tumoral ductal) (ALVES S, 2015).

O estudo dos mecanismos da doença é limitado pela dificuldade de se obter tecido em humanos e pela relativa ausência de modelos animais de pancreatite crônica (FELDMAN M et al, 2014).

No entanto, vários fatores têm sido relacionados com a doença. Na maioria dos estudos, o uso abusivo de álcool é responsável por dois terços de todos os casos de pancreatite crônica. $O$ álcool e seus metabólitos produzem estresse oxidativo e metabólico que levam a lesões acinares e, posteriormente, geram fibrose (ZHANG H et al, 2016).

Tem sido dada importância, ainda, às mutações genéticas que predispõem às pancreatites crônicas, apresentando relação com a pancreatite crônica hereditária, fibrose cística ou, também, com as pancreatites crônicas alcoólicas. Apesar de não estar claro que todas as diversas etiologias têm uma fisiopatologia idêntica, o resultado histológico final é semelhante (FELDMAN M et al, 2014).

A condução do doente portador de pancreatite crônica constitui, atualmente, um interessante desafio clínico. A gestão inclui abordagens médicas, endoscópicas e cirúrgicas com a necessidade de interação entre várias especialidades, exigindo uma ação multidisciplinar combinada (CZUL F et al., 2017).

Na maioria dos casos, inicia-se com tratamento conservador e, perante o agravamento ou a ausência de remissão dos sintomas, pondera-se uma abordagem mais invasiva (MARTINEZ JF et al., 2013).

Nesse contexto, observa-se que ainda hoje ocorre uma verdadeira construção das bases do conhecimento sobre a origem da doença e a busca de evidências a fim de estabelecer um consenso para 0 tratamento dessa afecção.

Assim sendo, é de extrema importância que os estudantes e profissionais da área sejam constantemente atualizados sobre o tema para que dessa forma o paciente seja mais bem assistido e apresente melhores respostas à terapêutica. Por conseguinte, tendo em vista esse cenário, o presente estudo teve como objetivo realizar uma revisão de literatura, entre o ano de 2014 e 2018, no que tange à fisiopatologia e ao tratamento da pancreatite crônica. 


\section{MÉTODOS}

Para a realização desta revisão de literatura, efetuada no período compreendido entre os meses de fevereiro a maio de 2018, foi realizada, por dois examinadores, uma consulta a livros e periódicos presentes na biblioteca da Faculdade Metropolitana da Amazônia (FAMAZ) e a artigos científicos selecionados através de buscas direcionadas nas bases de dados Medline e PubMed, publicados entre 2014 e 2018, em inglês, português ou espanhol.

Foram selecionados 02 livros disponíveis na biblioteca da FAMAZ. A busca nos bancos de dados foi realizada utilizando as terminologias cadastradas nos Descritores em Ciências da Saúde (DeCS). As palavras-chaves utilizadas na busca foram "pancreatite crônica", "fisiopatologia" e "terapia", e suas correspondentes em inglês "pancreatitis chronic", "physiopathology" e "therapy".

A busca nas bases de dados para os seguintes descritores: "pancreatite crônica" e "fisiopatologia" teve como resultado o número de 104artigos. Após a leitura dos resumos dos artigos, notou-se que alguns deles se repetiram nas diferentes bases e outros não preenchiam os critérios deste estudo, o que reduziu a amostra para 19 artigos. Em seguida foi feita a leitura completa dos artigos selecionados, totalizando05 artigos para a revisão.

A busca nas bases de dados para os seguintes descritores: "pancreatite crônica" e "terapia" apresentou ao todo os seguintes resultados: 617 artigos. Com a seguinte leitura dos resumos, observou-se que a repetição de diversos artigos em ambas as bases e foram descartados aqueles que eram restritos à experiências com animais e os que elucidavam apenas sobre as complicações, o que reduziu a amostra para 10 artigos.

\section{RESULTADOS E DISCUSSÕES}

\section{Fisiopatologia}

Diante da análise dos estudos dessa revisão, pôde-se observar que a fisiopatologia da pancreatite crônica ainda permanece mal compreendida. A pancreatite crônica alcoólica - que é a mais comum revelou-se como principal alvo de interesse da comunidade científica atualmente.

O álcool e seus metabólitos causam efeitos prejudiciais às células acinares pancreáticas, produzindo, sobretudo, um aumento patológico da sensibilidade destas células aos estímulos fisiológicos. Além do estresse oxidativo e metabólico causado às células acinares, essa substância provoca ainda lesão alcoólica às células ductais (ZHANG H et al, 2016). Além disso, o álcool e seus metabólitos parecem estimular as células estreladas do pâncreas (BYNIGERI RR et al, 2017).

A ativação das células estreladas pancreáticas ocorre tanto pelo álcool e seus metabólitos, como também, de forma indireta, por citoquinas induzidas por necrose celular, por isso alguns autores afirmam que a cascata inflamatória oxidativa desempenha um papel importante na progressão da fibrose pancreática. Esse mecanismo, no entanto, não justifica o desenvolvimento de pancreatite crônica em apenas $10 \%$ dos indivíduos que fazem uso abusivo de álcool (LIU FF et al, 2015). Além disso, é importante ressaltar que há autores que afirmam que a associação do etilismo com o tabagismo potencializa os efeitos tóxicos do etanol (FELDMAN M et al, 2014).

Há, ainda, outro mecanismo pelo qual o álcool pode levar à pancreatite crônica. O uso prolongado dessa substância leva à secreção de suco pancreático rico em proteínas e com concentração reduzida de bicarbonato. Essas características contribuem para a formação de precipitados de proteínas que podem calcificar e formar cálculos, produzindo mais lesão no parênquima pancreático e nos ductos (FELDMAN M et al, 2014).

Várias mutações foram identificadas, sugerindo que existe uma predisposição genética relativa para o desenvolvimento de pancreatite crônica: mutações no regulador da condutância transmembrana da fibrose cística (CFTR), no gene do tripsinogênio catiônico (gene PRSS1), no inibidor da serino-protease Kasal tipo 
1 (SPINK1, um inibidor de tripsina, antigamente clianiado de PSTI ou inibidor da tripsina secretória pancreática) e outras diversas também foram identificadas. (FELDMAN M et al, 2014).

CFTR é sigla utilizada para denominar tanto o gene quanto a proteína Cystic FibrosisTransmembrane Conductance Regulator. Quando há disfunção da proteína CFTR, o transporte transepitelial dos íons e água se modifica. No pâncreas, a consequência deste processo é de que a secreção de enzimas pancreáticas e de fluido rico em bicarbonato se torna prejudicada acarretando má digestão e absorção de nutrientes (ALVES S, 2015). A fibrose cística é uma doença autossômica recessiva causada por mutações que codificam o gene CFTR e, por esse motivo, existem várias pesquisas que buscam comprovar a associação entre fibrose cística e pancreatite crônica, mas os resultados geralmente divergem. (FELDMAN M et al, 2014).

\section{Tratamento}

Quanto ao tratamento da pancreatite crônica, sabe-se que o mesmo é essencialmente baseado no controle da dor e, quando necessária, na abordagem endoscópica e na intervenção cirúrgica. (FELDMAN M et al, 2014). No que diz respeito ao manejo da dor, alguns estudos consideram de suma importância o abandono do consumo de bebidas alcóolicas e do tabagismo (YANG D, FORSMARK C, 2017). Contudo, outros autores afirmam haver ainda uma compreensão incompleta desses mecanismos e que, apesar da cessação do tabagismo e da abstinência alcoólica serem largamente recomendadas no tratamento da pancreatite, ainda são necessárias evidências de alto nível para comprovar o real efeito dessas medidas na vida do paciente (SHABANZADEH DM, NOVOVIC S, 2017).

Ademais, recomenda-se o uso de analgésicos opióides (WANG D, 2017, p.40) e não opioides para os casos de dor abdominal crônica (SHARMA V et al, 2014). O uso inicial de paracetamol ou acetaminofeno com escalada para pregabalina e/ou tramadol seguido por oxicodona ou outros opióides fortes são razoáveis passos na escada do controle da dor na pancreatite crônica, preconizada pela Organização Mundial da Saúde. (SINGH VK, DREWES AM, 2017).

Após certo tempo de evolução da doença, por seu próprio padrão de evolução progressiva, é comum que haja o aparecimento de má absorção e de diabete mellitus associados, resultantes, respectivamente, da substituição gradativa do parênquima glandular secretor exócrino (ácinos e canalículos) e endócrino (ilhotas de Langerhans) por fibrose (ZATERKA S, EISIG JN, 2016). Ainda de acordo com Zaterka S (2016), a má absorção deve ser tratada pela administração de extratos pancreáticos, cuja dosagem varia em função do teor de gordura fecal, que pode ser avaliado após dieta padronizada, com o balanço de gordura perdida nas fezes.

A dieta do diabetes mellitus tipo III deve ter como prioridade a prevenção de eventos hipoglicêmicos, os quais podem ocorrer com frequência (DUGGAN SN et al, 2016). Recomenda-se, então, a aplicação de dietas com baixo teor de gorduras (FELDMAN M et al, 2014). Os pacientes devem ser educados, então, sobre a importância de manter um padrão alimentar regular com a inclusão de carboidratos ricos em amido e de não pular refeições. Ademais, devem ser educados sobre os sintomas e tratamento de hipoglicemia semelhante às orientações dadas aos portadores de diabetes mellitus tipo I (DUGGAN SN et al, 2016).

Estudos indicam que os antioxidantes podem reduzir um pouco a sensação dolorosa em pacientes com pancreatite crônica. Ainda assim, a relevância clínica desta pequena redução é incerta, e mais evidências são necessárias. (AHMED ALI U et al, 2014). Da mesma forma ocorre com as enzimas pancreáticas, apontadas como potenciais adjuvantes no tratamento da dor por pancreatite crônica, porém sem provas suficientes que apoiem o seu uso (SINGH VK, DREWES AM, 2017).

Feldman M (2014) afirma que em casos de obstrução ductal, problema muito comum nos pacientes com pancreatite crônica, existe a real necessidade de uma adequada intervenção endoscópica, a fim de melhorar a drenagem do ducto de Wirsung (DW). Geralmente esse quadro acontece por causa de uma pancreatolitíase. A colangio pancreatografia retrógrada endoscópica (CPRE) é considerada o tratamento de primeira linha no caso de um número pequeno de cálculos, com tamanhos abaixo de cinco $\mathrm{mm}$, localizados na cabeça ou no corpo do pâncreas. Já para os cálculos maiores que $5 \mathrm{~mm}$, recomenda-se a litotripsia 
extracorpórea por ondas de choque (LEOC), a qual é caracterizada como um procedimento seguro e efetivo inclusive para pacientes pediátricos com pancreatite crônica e que pode inclusive beneficiar o crescimento e o desenvolvimento dessas crianças. Contudo, é fato que nem sempre a dor responde à retirada da pedra.

Ainda segundo Feldman M (2014), a dor também pode ser em decorrência de uma estenose do DW. Sendo assim, a colocação de um stent pode dilatar o ducto e proporcionar um consequente alívio do sintoma. Ademais, em grande parte dos casos, é necessário fazer uma esfincterectomia do ducto pancreático para a devida colocação do stent. Todavia, em cerca de um terço dos pacientes, os sintomas recidivam depois de uma melhora clínica inicial. Para Ke N, et al. (2018), os procedimentos cirúrgicos no tratamento da pancreatite crônica podem ser agrupados em 3 categorias: procedimentos de drenagem, ressecção e mistos. O tipo de alterações morfológicas do pâncreas e o envolvimento de órgãos adjacentes influenciam na escolha da técnica. Evidências têm demonstrado que os procedimentos de Frey e Berne são as técnicas cirúrgicas mais efetivas, visto que apresentam os melhores resultados.

\section{CONSIDERAÇÕES FINAIS}

Diante da revisão efetuada, observou-se que a pancreatite crônica é uma doença associada a diferentes predisposições genéticas, gatilhos e uma via final comum semelhante, produzindo lesão e fibrose do parênquima pancreático, levando, por fim, à falência do órgão. No que se refere ao tratamento, notou-se que o mesmo tem como um de seus principais objetivos o controle da dor, mas muitas das recomendações para este fim, como a cessação do consumo de bebida alcoólica, amplamente divulgada, ainda são questionadas por alguns estudos, diante da escassez de evidências que comprovem grande parte de tais medidas. Fato este que se repete quando se analisa, por exemplo, a abordagem cirúrgica, pois ainda não existe um consenso sobre o melhor momento para a sua realização. Por conseguinte, percebe-se, então, a relevante necessidade de que haja novas pesquisas sobre o assunto a fim de se esclarecer, de forma consistente, a fisiopatologia da pancreatite crônica e de que maneira cada parte da terapêutica influencia na melhora clínica do paciente portador dessa afecção.

\section{REFERÊNCIAS}

1. AHMED ALI U, et al. Antioxidantes para reduzir dor em pancreatite crônica. Cochrane. Disponível em: http://www.cochrane.org/pt/CD008945/antioxidantes-para-reduzir-dor-empancreatite-cronica. Acesso em: 11 mai. 2018.

2. ALVES S. Etiologia e Patogênese da Pancreatite Crônica no Adulto. Dissertação (Medicina) - Instituto de Ciências Biomédicas Abel Salazar. Universidade do Porto, Porto, 2015.

3. BYNIGERI RR, et al. Pancreatic stell at cell: Pandora's box for pancreatic disease biology. World Jornal of Gastroenterology, $2017 ; 23$ (3): 382-405.

4. CZUL F, et al. Update on chronic pancreatitis: review article. Revista de Gastroenterología del Peru, Lima, $2017 ; 37$ (2): $146-155$.

5. DUGGAN SN, et al. The Nutritional Management of Type 3c (pancreatogenic) Diabetes in Chronic Pancreatitis. European Journal of Clinical Nutrition, London, 2016; 71 (1): 1-6.

6. FELDMAN M, et al. Tratado Gastrointestinal e Doenças do Fígado: fisiopatologia/diagnóstico/tratamento. 9. ed. São Paulo: Elsevier Editora Ltda, 2014.

7. LIU FF, et al. The role of oxidative inflammatory cascade on pancreatic fibrosis progression in mice induced by DBTC plus ethanol. Zhongguo Ying Yong Sheng Li Xue Za Zhi, Pequim, 2015; 31 (5): 477-480.

8. MARTÍNEZ JF, et al. The Spanish Pancreatic Club recommendations for the diagnosis and treatment of chronic pancreatitis: part 1 (diagnosis). Pancreatology, Philadelphia, 2013; 13 (1): 8-17.

9. KE N, et al. Earlier surgery improves outcomes from painful chronic pancreatitis. Medicine, Hagerstown, 2018 ; 97 (19): 1-7.

10. NEVES S, RIBEIRO Al. Tratamento da Pancreatite Crônica: Abordagem Endoscópica vs. Cirúrgica. Dissertação. (Medicina) Instituto de Ciências Biomédicas Abel Salazar. Universidade do Porto, Porto, 2014.

11. SHABANZADEH DM, NOVOVIC S. Alcohol, smoking and benign hepato-biliary disease. Best practice \& research: Clinical Gastroenterology, Amsterdam, 2017; 31 (5): 519-527.

12. SINGH VK, DREWES AM. Medical Management of Pain in Chronic Pancreatitis. Digestive diseases and sciences, New York, 2017; 62 (7): 1721-1728.

13. WANG D. Opioid Medications in the Management of Chronic Abdominal Pain. Current pain and headache reports, Philadelphia, 2017; 21 (9): 40.

14. YANG D, FORSMARK CE. Chronic pancreatitis. Current Opinion in Gastroenterology, Boston, $2017 ; 33$ (5): $396-403$.

15. ZATERKA S, EISIG JN. Tratado de Gastroenterologia: da graduação à pós graduação. 2. Ed. São Paulo: Atheneu, 2016.

16. ZHANG H, et al. Pathophysiology of chronic pancreatitis induced by dibutyltin dichloride joint ethanol in mice. World Jornal of Gastroenterology, Long Beach, 2016; 22 (10): 2960-2970. 